\title{
An Upper Bound for the Minimum Diameter of Integral Point Sets
}

\author{
Heiko Harborth, Arnfried Kemnitz, and Meinhard Möller \\ Diskrete Mathematik, Technische Universität Braunschweig, \\ W-3300 Braunschweig, Federal Republic of Germany
}

\begin{abstract}
For all $n>d$ there exist $n$ points in the Euclidean space $E^{d}$ such that not all points are in a hyperplane and all mutual distances are integral. It is proved that the minimum diameter of such integral point sets has an upper bound of $2^{\log n \log \log n}$.
\end{abstract}

\section{Introduction}

Sets of $n$ points in the Euclidean space $E^{d}$, not all in a hyperplane, do exist for all $n>d$ such that all mutual distances are integral. Proofs are published in [10] and [12] for plane concyclic sets, and in [3] and [4] for sets in higher dimensions, in addition in [4] no three points are allowed to be collinear.

First upper bounds for the minimum diameter $k(d, n)$ of such integral sets of $n$ points in $E^{d}$ are given in [3] where the diameter means the largest distance of two points of the set. These bounds are of order $2^{n}$ for any fixed dimension.

In this note we construct concyclic integral point sets which improve the upper bound of $k(2, n)$ to $2^{c \log n \log \log n}$, that is $n^{c \log \log n}$. Then these constructions are used to find corresponding results for $d \geq 3$. Some exact values are presented for small numbers of points.

\section{Construction of Plane Integral Point Sets}

We obtain a concyclic integral point set by appropriate rotations of an equilateral triangle around its center.

Theorem 1. On a circle of diameter $\frac{2}{3} R \sqrt{3}$ there exist $n=3 \tau(R)=3 \prod_{j=1}^{m}\left(v_{j}+1\right)$ points with pairwise integral distances if $R=\prod_{j=1}^{m} p_{j}^{v_{j}}$ for any $m$ distinct prime numbers $p_{j} \equiv 1(\bmod 3)$. 
Proof. We consider the Euclidean quadratic number field $K(\rho)$ with $\rho=$ $(-1+\sqrt{-3}) / 2$. In $K(\rho)$ the rational primes $p \equiv 1(\bmod 3)$ have a unique prime factorization $p=\omega \bar{\omega}$ where $\omega=a+b \rho$ and $\bar{\omega}=a+b(-1-\sqrt{-3}) / 2=a-$ $b-b \rho$ are conjugate primes of $K(\rho)$ with rational integers $a$ and $b$. For any element $\alpha=a+b \rho$ of $K(\rho)$ its norm is $\alpha \bar{\alpha}=|\alpha|^{2}=a^{2}-a b+b^{2}$ (see Chapter 12 of [6], or [7]).

For each of the $\tau(R)$ divisors of $R$,

$$
\prod_{j=1}^{m} p_{j}^{u_{j}}=\prod_{j=1}^{m} \omega_{j}^{u_{j}} \bar{\omega}_{j}^{u_{j}}, \quad 0 \leq u_{j} \leq v_{j}
$$

and with

$$
\begin{gathered}
\eta_{3 h}=\prod_{j=1}^{m} \omega_{j}^{v_{j}+u_{j}} \bar{\omega}_{j}^{v_{j}-u_{j}}, \quad \eta_{3 h-1}=\rho \eta_{3 h}, \\
\eta_{3 h-2}=\rho^{2} \eta_{3 h}, \quad 1 \leq h \leq \tau(R),
\end{gathered}
$$

we consider the vertices $\xi_{s}, 1 \leq s \leq 3 \tau(R)$, of $\tau(R)$ equilateral triangles with their centers in the origin and with circumradius $\frac{1}{3} R \sqrt{3}$, where

$$
\xi_{3 h-k}=\frac{\sqrt{3}}{3 R} \eta_{3 h-k}^{2}, \quad k=0,1,2, \quad 1 \leq h \leq \tau(R) .
$$

All vertices $\xi_{s}$ are pairwise distinct since the prime factorization in $K(\rho)$ is unique.

For

$$
\eta_{s}=\rho^{k_{s}} \eta_{3 h}=x_{s}+y_{s} \rho, \quad 0 \leq k_{s} \leq 2, \quad 1 \leq s \leq 3 \tau(R),
$$

and with rational integers $x_{s}, y_{s}$ we conclude

$$
\begin{aligned}
\left|\eta_{s}\right|^{2} & =\eta_{s} \bar{\eta}_{s}=x_{s}^{2}-x_{s} y_{s}+y_{s}^{2}=\prod_{j=1}^{m} \omega_{j}^{2 v_{j}} \bar{\omega}_{j}^{2 v_{j}} \\
& =\prod_{j=1}^{m} p_{j}^{2 v_{j}}=R^{2}
\end{aligned}
$$

This implies that all points $\xi_{s}$ are concyclic with radius $(R / 3) \sqrt{3}$.

We use

$$
\eta_{s}^{2}=R^{2}-\frac{3}{2} y_{s}^{2}+i \sqrt{3}\left(x_{s} y_{s}-\frac{1}{2} y_{s}^{2}\right)
$$

with $i=\sqrt{-1}$ to determine the distance of any two points $\xi_{s}$ and $\xi_{t}, 1 \leq s$, $t \leq 3 \tau(R)$. We obtain

$$
\begin{aligned}
R^{2}\left|\xi_{t}-\xi_{s}\right|^{2} & =\frac{1}{3}\left|\eta_{t}^{2}-\eta_{s}^{2}\right|^{2} \\
& =\frac{1}{3}\left|\frac{3}{2}\left(y_{s}^{2}-y_{t}^{2}\right)+i \sqrt{3}\left(\frac{1}{2}\left(y_{s}^{2}-y_{t}^{2}\right)+x_{t} y_{t}-x_{s} y_{s}\right)\right|^{2} \\
& =\left(y_{s}^{2}-y_{t}^{2}\right)^{2}+\left(y_{s}^{2}-y_{t}^{2}\right)\left(x_{t} y_{t}-x_{s} y_{s}\right)+\left(x_{t} y_{t}+x_{s} y_{s}\right)^{2} \\
& =\left(y_{s}^{2}-y_{t}^{2}\right)\left(R^{2}-x_{s}^{2}-\left(R^{2}-x_{t}^{2}\right)\right)+\left(x_{t} y_{t}-x_{s} y_{s}\right)^{2} \\
& =\left(x_{t} y_{s}-x_{s} y_{t}\right)^{2}
\end{aligned}
$$


which implies

$$
\left|\xi_{t}-\xi_{s}\right|=\frac{1}{R}\left|x_{t} y_{s}-x_{s} y_{t}\right|
$$

Since

$$
\begin{aligned}
\eta_{s} \bar{\eta}_{t} & =\left(x_{s}+y_{s} \rho\right)\left(x_{t}+y_{t} \bar{\rho}\right) \\
& =x_{s} x_{t}-x_{s} y_{t}+y_{s} y_{t}+\rho\left(x_{t} y_{s}-x_{s} y_{t}\right)
\end{aligned}
$$

all distances $\left|\xi_{t}-\xi_{s}\right|$ are integers if $\eta_{s} \bar{\eta}_{t}$ and thus $x_{t} y_{s}-x_{s} y_{t}$ is divisible by $R$. We obtain

$$
\begin{aligned}
\eta_{s} \bar{\eta}_{t} & =\rho^{k_{s}} \bar{\rho}^{k_{t}} \prod_{j=1}^{m} \omega_{j}^{v_{j}+u_{j}} \bar{\omega}_{j}^{v_{j}-u_{j}} \prod_{j=1}^{m} \bar{\omega}_{j}^{v_{j}+w_{j}} \omega_{j}^{v_{j}-w_{j}} \\
& =\rho^{k_{s}-k_{t}} \prod_{j=1}^{m} \omega_{j}^{2 v_{t}+u_{j}-w_{j}} \bar{\omega}_{j}^{2 v_{j}-u_{j}+w_{j}} \\
& =\rho^{k_{s}-k_{t}} \prod_{j=1}^{m} p_{j}^{v_{j}} p_{j}^{v_{j}-\left(u_{j}+w_{j}-2 q_{j}\right)} \omega_{j}^{2\left(u_{j}-q_{l}\right)} \bar{\omega}_{j}^{2\left(w_{j}-q_{j}\right)} \\
& =R(A+B \rho),
\end{aligned}
$$

where $q_{j}=\min \left(u_{j}, w_{j}\right)$, and $A$ and $B$ are rational integers since the exponents of the last product are nonnegative. Therefore $R$ divides $\eta_{s} \bar{\eta}_{t}$, and the proof of Theorem 1 is complete.

\section{An Upper Bound for $k(2, n)$}

We use Theorem 1 to get the following upper bound for the minimum diameter $k(2, n)$.

\section{Theorem 2.}

$$
k(2, n)<2^{c \log n \log \log n}, \quad c=\text { constant }
$$

Proof. We choose the first $m$ prime numbers $p_{j} \equiv 1(\bmod 3)$ and $v_{j}=1$ for $1 \leq j \leq m$ to get, from Theorem 1 ,

$$
k(2, n)<\frac{2}{3} \sqrt{3} \prod_{j=1}^{m} p_{j} \quad \text { and } \quad n=3 \cdot 2^{m}
$$

We use the well-known results from number theory (see, for example, Chapters 1 and 22 of [6]) that

$$
\prod_{j=1}^{m} p_{j}<\prod_{j=1}^{c_{1} m} q_{j}, \quad q_{j}=j \text { th prime number, } \quad q_{j}<c_{2} j \log j
$$


and

$$
\prod_{q \leq x} q<c_{3}^{x}, \quad q \text { is a prime number, }
$$

with constants $c_{1}, c_{2}, c_{3}$. We get

$$
k(2, n)<\frac{2}{3} \sqrt{3} \prod_{q \leq c_{1} c_{2} m \log \left(c_{1} m\right)} q<2 c_{3}^{c_{1} c_{2} m \log \left(c_{1} m\right)}
$$

Together with $m<c_{4} \log n$ and constants $c, c_{4}, c_{5}$ it follows that

$$
k(2, n)<c_{3}^{c s \log n \log \log n}=2^{c \log n \log \log n} .
$$

\section{Higher Dimensions}

The construction used for Theorem 1 also implies an upper bound $k(d, n)$ in general.

\section{Theorem 3.}

$$
k(d, n)<2^{c \log n \log \log n}, \quad c=\text { constant }
$$

Proof. We choose, corresponding to Theorem $1, n-d+2$ points with pairwise integral distances on a circle of radius $R \sqrt{3}$. The remaining $d-2$ points are chosen as follows: We consider a regular $(d-2)$-simplex of side length $R$ in that subspace orthogonal to the plane of the circle so that one of its vertices is the center point of the circle. Then all other $d-2$ vertices of this $(d-2)$-simplex have distance $2 R$ to all points of the circle. The diameter of these $n$ points is less than $2 R \sqrt{3}$, and Theorem 2 completes the proof.

For $d \geq 3$ we know other constructions of integral point sets with smaller diameters, for example, if several two-dimensional circular point sets of Theorem 1 are combined, however, no construction is known to us which implies a smaller order of magnitude.

\section{Exact Values for Small Point Sets in the Plane}

No reasonable lower bounds are known in general. For the plane the following exact values of $k(2, n)$ are determined with the aid of a computer:

$$
k(2, n)=1,4,7,8,17,21,29 \quad \text { for } n=3,4, \ldots, 9
$$



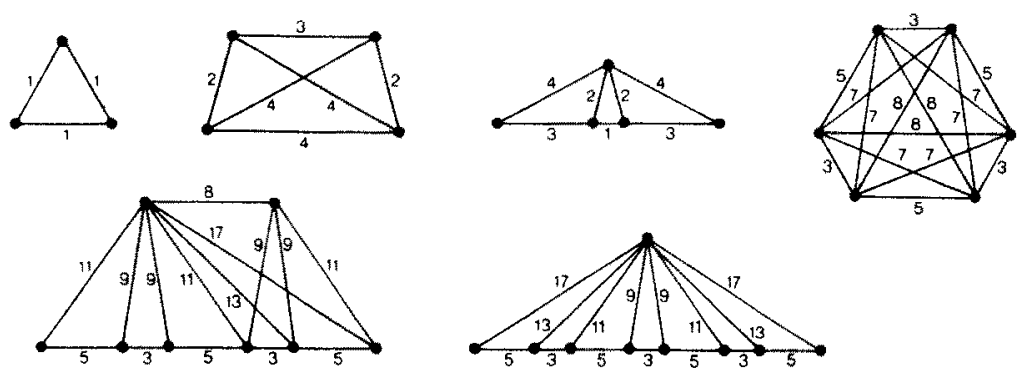

Fig. 1

see Fig. 1 for examples, where the example for $n=7$ follows from that for $n=8$ by deletion of one of the two points at distance 21 .

The construction in the proof of Theorem 1 always gives concyclic integral point sets. In general, we have determined by computer, for $n \leq 9$, the smallest integral point sets without any three being collinear; see Fig. 2 for those which are not in Fig. 1 (see also [11]). An example for $n=8$ follows from that for $n=9$ by deletion of one point.

For $n>9$ upper bounds follow from constructions by Theorem 1. Some upper bounds, for example for $k(2,12)$ and $k(2,24)$, are already given in [1].

If no three points are collinear and, moreover, no four concyclic, then the question for integral point sets is a so-called Erdös problem (see also Section D20 of [2]). For $n \geq 7$ even the existence of such special integral point sets is unknown. The smallest diameters for $n=4,5$, and 6 are 8,73, and 174 (see [3], [4], and [9]).

Another modified problem is proposed in [8]: to find integral point sets with the minimum sum of all distances.

For $d \geq 3$ nearly no examples of minimum integral point sets are known. Some first results can be found in [5].
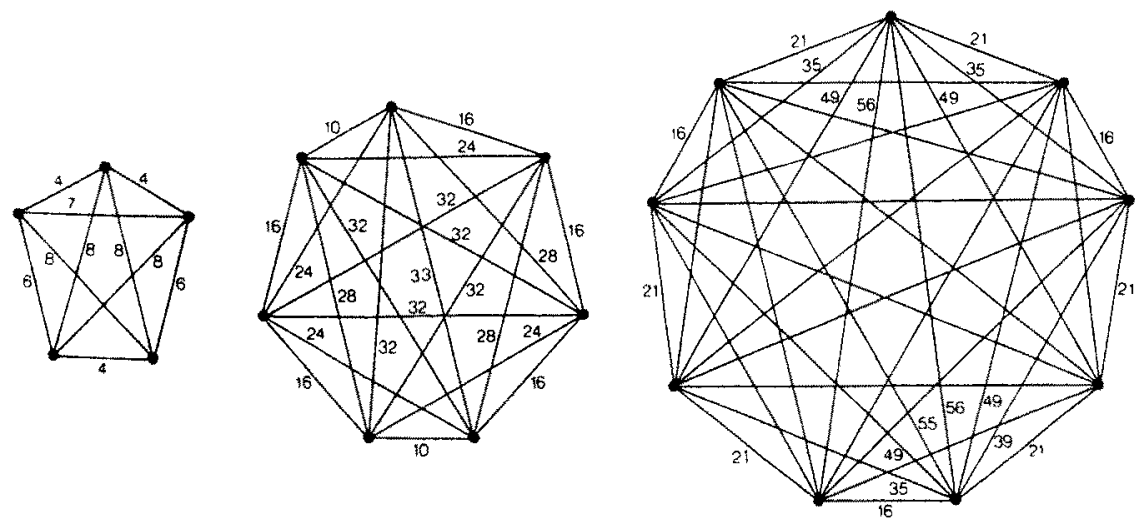

Fig. 2 


\section{References}

1. N. Anning, Relating to a geometric representation of integral solutions of certain quadratic equations, Amer. Math. Monthly 22 (1915), 321.

2. R. K. Guy, Unsolved Problems in Number Theory, Springer-Verlag, New York, 1981.

3. H. Harborth and A. Kemnitz, Diameters of integral point sets, in Intuitive Geometry (Proc. Conf. Siófok (Hungary), 1985), pp. 255-266, Colloquia Mathematica Societatis János Bolyai, Vol. 48, North-Holland, Amsterdam, 1987.

4. H. Harborth and A. Kemnitz, Integral representations of graphs, in Contemporary Methods in Graph Theory (eds. R. Bodendiek and R. Henn), pp. 359-367, BI, Mannheim, 1990.

5. H. Harborth and L. Piepmeyer, Point sets with small integral distances, The Victor Klee Festschrift, pp. 319-324, DIMACS Series in Discrete Mathematics and Theoretical Computer Science, Vol. 4, 1991.

6. G. H. Hardy and E. M. Wright, An Introduction to the Theory of Numbers, 4th edition, Oxford University Press, Oxford, 1975.

7. H. Hasse, Vorlesungen über Zahlentheorie, 2nd edition, Springer-Verlag, Berlin, 1964.

8. J. H. Jordan, Lesser-known integer polygons, Manuscript, 1991.

9. A. Kemnitz, Punktmengen mit ganzzahligen Abständen, Habilitationsschrift, Braunschweig, 1988.

10. D. N. Lehmer, Rational triangles, Ann. of Math. (2) 1 (1899/1900), 97-102.

11. M. Möller, Ganzzahlige Darstellungen von Graphen in der Ebene, Dissertation, Braunschweig, 1990.

12. A. Müller, Auf einem Kreis liegende Punktmengen ganzzahliger Entfernungen, Elem. Math. 8 (1953), 37-38.

Received July 18, 1991, and in revised form November 15, 1991. 